\title{
Multifunctional activity of graphene oxide-based nanoformulation against the disease vector, Aedes aegypti
}

\author{
Devaj Gupta \\ DPS International, Saket, New Delhi-110057, India \\ Roopa Rani Samal* \\ Department of Zoology, Acharya Narendra Dev College, University of Delhi, Kalkaji, \\ New Delhi-110019, India \\ Drashya Gautam \\ Department of Chemistry, Acharya Narendra Dev College, University of Delhi, Kalkaji, \\ New Delhi-110019, India \\ Sunita Hooda \\ Department of Chemistry, Acharya Narendra Dev College, University of Delhi, Kalkaji, \\ New Delhi-110019, India

\section{Sarita Kumar} \\ Department of Zoology, Acharya Narendra Dev College, University of Delhi, Kalkaji, \\ New Delhi-110019, India \\ *Corresponding author. Email: rupasamal29@gmail.com
}

\section{How to Cite}

Gupta, D. et al. (2021). Multifunctional activity of graphene oxide-based nanoformulation against the disease vector, Aedes aegypti. Journal of Applied and Natural Science, 13(4), 1265 - 1273. https://doi.org/10.31018/jans.v13i4.3018

\begin{abstract}
Aedes aegypti management is a global concern due to the absence of medication and effective vaccines. The pesticidemediated health hazards and rising insecticide resistance in mosquitoes have aggravated the issues. As graphene Oxide (GO)-based nanoformulations are considered a novel mosquito management strategy; the present investigation evaluated the efficacy of GO-based nanoformulations conjugated with malathion (ML) and endosulphan (EN) against Ae. aegypti. The GO was synthesised by Hummers' method and was confirmed by UV-visible spectral analysis. The GO-ML and GO-EN binary mixtures (1:1 and 1:2) were assayed for toxic potential against mosquito larvae as per WHO protocol and the dead larvae were scrutinized for morphological deformations/abnormalities. The contact irritancy potential of GO nanoformulations was also evaluated against adult $A e$. aegypti. The UV-visible spectrum of GO showed a narrow and high peak at $\sim 300 \mathrm{~nm}$ corresponding to an $\mathrm{n}-\pi^{*}$ plasmon peak. The GO-insecticide binary mixtures augmented the ML and EN toxicity by $80.43 \%$ and $6.43-$ fold, respectively. The GO-ML mixture-exposed larvae revealed cuticular deposition of black soot while larvae exposed to GO-EN exhibited disintegrated gut viscera. GO-insecticide combinations increased flights in Ae. aegypti denoting irritant potential. The effectual toxic, abrasive and irritant activity of GO-insecticide nanoformulations recommends developing graphene-based toxicants for mosquito management.
\end{abstract}

Keywords: Aedes aegypti, Contact irritancy, Graphene oxide, Insecticide, Larvicidal, Nanoformulation

\section{INTRODUCTION}

Insect pests and vector management has become a global problem nowadays. Among several control interventions, the use of chemicals is more frequent owing to their quick action and effective results. Nevertheless, the application of pesticides not only causes various environmental, social and health hazards but also results in increasing insecticide resistance in the target organism. Moreover, a high percentage of the chemical is lost via degradation, photolysis, and volatilization, imparting less effective actions (Monteiro et al., 2021). Consequent use of increasing dosages at much higher frequency aggravates the problems associated with the insecticides. Thus, nanopesticides are receiving increasing attention as a new strategy for insect vector and pest management.

Nanopesticide formulations are regarded as comparatively beneficial than conventional insecticide formulations as they require reduced molar contents of the pesticide, are relatively more durable, have better solubility and exhibit higher efficacy against the target (Lu 
et al., 2021). In addition, their small surface-to-volume ratio gives advantageous and improved chemical and physical features in comparison to their bulk counterparts (Samadi et al., 2017). It is believed that nanopesticides may provide an efficient solution for the current overuse of pesticides globally. It is hypothesized that the synergistic action of nanomaterial and pesticides could mitigate these impacts resulting in a more efficient and safer alternative (Jampilek et al., 2020; Zhang et al., 2021).

Graphene oxide (GO) is a unique material which is composed of a monolayer of $s p^{2}$ bonded carbon atoms and possesses a large surface area resulting in high thermal, mechanical and electronic properties (Samadi et al., 2017). GO is considered one of the appropriate nanomaterials which commendably responds to the environmental stimuli, can absorb the pesticide particles and releases pesticide macromolecules in controlled amount (Lu et al., 2021). Hence, GO can be used to formulate effectual nanopesticides against insect pests and vectors.

Aedes aegypti is a widespread disease vector of Chikungunya, yellow fever, dengue and Zika. The increased number of disease incidences throughout the world has augmented fatalities and health problems in human beings. In addition, different formulations of chemical insecticides used to control this vector have aggravated these issues. The promising use of nanotechnology in mosquito management can help in mitigating the harmful effects of currently used pesticides. Attempts have been made to use synergistic mixtures of $\mathrm{GO}$ and insecticides against a few organisms. Combinations of GO with chlorpyrifos, pyridaben and $\beta$ cyfluthrin were found to exhibit higher contact toxicity against spider mites; Tetranychus urticae and T. truncatus; revealing 1.78, 1.75 and 1.50-fold and 1.55, 1.56 and 1.77-fold more impact, respectively (Wang et al., 2019a). The efficient synergistic activity of GO with $\beta$ cyfluthrin, monosultap and imidacloprid has also been observed against Asian corn borer resulting in 2.10, 1.51 and 1.83-fold higher contact toxicities than the respective individual insecticides (Wang et al., 2019b). However, the novelty of this technology is yet to be investigated in detail against mosquito populations in comparison to lone insecticides.

Malathion is an organophosphate which inhibits acetylcholineesterase in the nerve synaptic region of the target insect; whereas endosulphan is an organochlorine which inhibits gamma-Aminobutyric acid (GABA) chloride ionophore complex (Dixit et al., 2021). Thus, present investigation evaluated the efficacy of nanoformulations formed with $\mathrm{GO}$ in combination with malathion and endosulphan separately, against the Indian strain of $\mathrm{Ae}$. aegypti. Synergistic combinations of insecticides-GO were evaluated for their larvicidal potential, morphology aberration effects and contact irri- tancy against $A e$. aegypti with a proposition that graphene oxide-insecticide nanoformulations can result in highly effective insecticidal action.

\section{MATERIALS AND METHODS}

\section{Synthesis of graphene oxide nanomaterial}

The graphene oxide was synthesized by the Hummers' method (Chen et al., 2013) using graphite powder. The mixture of $0.5 \mathrm{~g}$ of $\mathrm{NaNO}_{3}$ and $1.0 \mathrm{~g}$ of graphite powder was added to the concentrated $\mathrm{H}_{2} \mathrm{SO}_{4}(23 \mathrm{~mL})$ in a 500 $\mathrm{mL}$ beaker kept in an ice bath at $5{ }^{\circ} \mathrm{C}$ (Gautam et al., 2020). The mixture was stirred continuously for $5 \mathrm{~min}$, followed by the gradual and slow addition of $\mathrm{KMnO}_{4}$ $(3 \mathrm{~g})$. The rate of addition was carefully monitored to prevent the rise in temperature $>15^{\circ} \mathrm{C}$. After $2 \mathrm{~h}$, the beaker was taken out from the ice bath and as soon as the temperature rose to $35{ }^{\circ} \mathrm{C}$, the mixture was constantly stirred for the next $30 \mathrm{~min}$. Subsequently, deionised water was added increasing the mixture temperature rapidly to $98{ }^{\circ} \mathrm{C}$. This mixture was against stirred for next $30 \mathrm{~min}$ to maintain the rising temperature. The reaction was terminated by the addition of $\mathrm{H}_{2} \mathrm{O}_{2}(10 \%$ $\mathrm{v} / \mathrm{v}, 10 \mathrm{~mL}$ ) and deionised water resulting in a yellow colour product which was then subjected to vacuum filtration forming a brownish-yellow precipitate. The precipitate was separated and rinsed $5 \mathrm{X}$ with $\mathrm{HCl}(5 \%$, $200 \mathrm{~mL}$ ) and warm deionised water to remove the unreacted $\mathrm{Mn}^{2+}$ and acid. The graphene oxide nanopowder was obtained by drying the precipitate in an oven at $60{ }^{\circ} \mathrm{C}$ for $12 \mathrm{~h}$.

\section{Confirmatory analysis of the nanomaterial synthesis: UV-visible spectral analysis}

The optimal synthesis of nanomaterial was confirmed by UV-visible spectral analysis. The synthesis was tracked through intensity measured by UV-visible spectrophotometer (UV-1800 Shimadzu, Japan). Periodically, $1 \mathrm{~mL}$ aliquots of the sample were dispersed in $2 \mathrm{~mL}$ of double-distilled water and scanned at 200 to $900 \mathrm{~nm}$ wavelength with $1 \mathrm{~nm}$ resolution.

\section{Insecticides used in the study}

The susceptibility of $A e$. aegypti was tested against two classes of insecticides; an organophosphate (Malathion - ML) and an organochlorine (Endosulphan - EN). These insecticides were obtained from M/s SigmaAldrich, India. Their structural formulae, chemical name and mode of action are presented in Table 1.

\section{Preparation of nanomaterials-insecticide formulation}

Each insecticide (ML and EN) was added to the nanomaterial (GO) in the ratio of $1: 1$ and $1: 2$. The mixture of one part of the $\mathrm{GO}$ dispersed in $2 \mathrm{~mL}$ of acetone 0.1 $\mathrm{mL}$ of tween 80 solution, and one/two parts of insecticide (ML/EN), was ultrasonicated in water $(100 \mathrm{~mL})$ 
Table 1. Insecticides used in the study

\begin{tabular}{|c|c|c|c|c|c|}
\hline Class & Insecticide & IUPAC name & Structure & Mode of Action & \%Purity \\
\hline $\begin{array}{l}\text { Malathion } \\
(\mathrm{ML})\end{array}$ & Organophosphate & $\begin{array}{l}\text { Diethyl 2- } \\
\text { [(dimethoxyphosph } \\
\text { orothioyl)sulfanyl] } \\
\text { butanedioate }\end{array}$ & & $\begin{array}{l}\text { Acetylcholinester- } \\
\text { ase inhibitor }\end{array}$ & $99 \%$ \\
\hline $\begin{array}{l}\text { Endosulphan } \\
\text { (EN) }\end{array}$ & Organochlorine & $\begin{array}{l}6,7,8,9,10,10- \\
\text { Hexachloro- } \\
1,5,5 a, 6,9,9 a- \\
\text { hexahydro- } 6,9- \\
\text { methano-2,4,3- } \\
\text { benzodioxathiepine } \\
\text {-3-oxide }\end{array}$ & & $\begin{array}{l}\text { Inhibits gamma- } \\
\text { Aminobutyric ac- } \\
\text { id (GABA) chloride } \\
\text { ionophore complex }\end{array}$ & $99 \%$ \\
\hline
\end{tabular}

and stirred for $24 \mathrm{~h}$ in the dark. The compositions were then dissolved in $100 \mathrm{~mL}$ of autoclaved water. Serial dilutions were prepared with distilled water for investigations.

\section{Culture establishment of Ae. aegypti}

Pure strain of dengue fever mosquito, Ae. aegypti has been maintained in the rearing laboratory of Acharya Narendra Dev College, University of Delhi, India, under controlled conditions of $28 \pm 1{ }^{\circ} \mathrm{C}, 80 \pm 5 \% \mathrm{RH}, 14 \mathrm{~L} / 10$ D photoregime (Samal and Kumar, 2018). Adults were fed on sugary juice of water-soaked raisins, while females were provided with infrequent blood meals. The eggs were allowed to hatch in dechlorinated water. The larvae were then reared in water-filled trays added with powdered dog biscuits and yeast (3:1). The pupae formed were kept in the clothed cages for adult emergence (Samal et al., 2020; Gupta et al., 2021).

\section{Effect of GO and GO-Insecticide formulations on Ae. aegypti}

The insecticide and GO nanomaterials were mixed in different ratios $(1: 1$ and $1: 2$ by $v / v)$. These binary mixtures were used for investigating their impacts on the survival, morphology and irritancy of $A e$. aegypti.

\section{Larval toxicity bioassay}

Larvicidal bioassay on Ae. aegypti was performed in compliance with the WHO procedure (World Health Organization, 2016). Acetone was used as a solvent for GO-insecticide formulations. Batches of 20 early fourth instar larvae were transferred to $199 \mathrm{~mL}$ distilled water and $1 \mathrm{~mL}$ of the desired formulation of insecticide alone or the insecticides mixed with GO nanomaterial. Each dilution was repeated four times concurrently. The desired formulation was replaced by $1 \mathrm{~mL}$ of acetone for controls. After $24 \mathrm{~h}$, dead and moribund larvae were recorded as \%larval mortality. The test was discarded and repeated in case of more than $>10 \%$ pupation and $>20 \%$ larval mortality while the larval mortality was corrected as per Abbott's formula if control mortality ranged from 5\%-20\% (Abbott et al., 1925).
$\%$ Corrected mortality $=(\%$ Test mortality $-\%$ Control mortality) / (100 - \% Control mortality) x 100

Lethal concentrations (LC) causing 50\% and $90 \%$ larval mortality ( $\mathrm{LC}_{50}$ and $\mathrm{LC}_{90}$, respectively) along with the 95\% confidence intervals $(\mathrm{Cl})$ were computed from a log dosage-probit mortality regression line using computer software programs SPSS 22.0. Other statistical parameters; slope, standard error (SE), regression coefficient (RC) and chi square values were also computed. The $\mathrm{LC}_{50}$ values were considered significantly different if their $95 \% \mathrm{Cl}$ were not overlapped (Samal and Kumar, 2021). The co-toxicity coefficient, expected mortalities and synergistic factors were calculated as per formulae given below (Kalyanasundaram and Das, 1985; Trisyono and Whalon, 1999).

CTC (Co-toxicity coefficient) $=$ Observed $\%$ mortality -

Expected \% mortality / Expected \% mortality X 100

CTC value $\geq 20$ indicate synergism;

CTC value $\leq(-) 20$ indicate antagonism;

CTC intermediate value of $\leq(-) 20$ to $\geq 20$ indicate additive effect

Synergistic factor (SF) = Toxicity of insecticide alone/ Toxicity of insecticide with nanopcomposites

Values of SR $>1$ indicate synergism .....Eq. 3

Value of $S R<1$ indicate antagonism

\section{Morphological aberrations in larvae}

The dead larvae were scrutinized for any deformations and abnormalities in their external features to assess the effect of GO and GO-insecticide synergized combinations. The aberrations were photographed using with Magnus MIPS Camera, Olympus India Pvt. Limited.

\section{Contact irritancy against adult females}

Whatman filter paper circles were separately saturated with GO-insecticide formulations of $0.01 \%$ diluted with ethanol. The circles were dried and placed on the glass plates, over which was kept an inverted glass cone with a hole on the top. A 3-day old unfed female mos- 
quito was released into the cone and the hole was plugged with a cotton swab to prevent the mosquito escape. Post 3 minutes settling time, the time taken for the first flight and the total number of flights were recorded in the next 15 minutes. Each formulation was replicated 10 times. Controls assays were run with ethanol-impregnated Whatman filter papers. Similar assays were held with the blood-fed mosquitoes.

\section{RESULTS}

\section{UV-visible spectral analysis of Graphene oxide nanomaterial}

The UV-vis spectrum of the formulated $1 \%$ graphene oxide nanomaterial is presented in Fig 1. A characteristic narrow and high peak can be observed at $\sim 300 \mathrm{~nm}$, corresponding to an $n-\pi^{*}$ plasmon peak.

\section{Larvicidal bioassay with insecticides}

The larvicidal potentialities of malathion and endosulphan evaluated against early fourth instar of $A e$. aegypti displayed 19.06-fold higher toxicity of malathion than endosulphan (Table 2). The larval subjection to malathion resulted in $\mathrm{LC}_{50}$ value of $0.046 \mathrm{mg} / \mathrm{L}$ and $\mathrm{LC}_{90}$ value of $0.877 \mathrm{mg} / \mathrm{L}$. The non-overlapped $\mathrm{d}-\mathrm{m}-\mathrm{r}$ lines of malathion and endosulphan at $24 \mathrm{~h}$ denotes significant differences at $\mathrm{LC}_{50}$ level of dose (Fig. 2) $(p<$ 0.05).

\section{Synergistic larvicidal studies with $\mathrm{GO}+$ insecti-} cides (1:1 and 1:2)

\section{GO + Malathion (1:1 and 1:2)}

Addition of equal parts of malathion and GO increased the larvicidal efficacy of malathion after $24 \mathrm{~h}$ by 2.71fold at $L C_{50}$ level. Increasing the malathion proportion in the mixture (GO:ML::1:2) increased the toxic effects by 5.11-fold, demonstrating $80.43 \%$ higher larval mortality at median lethal dosage. The CTC (\% suppression)

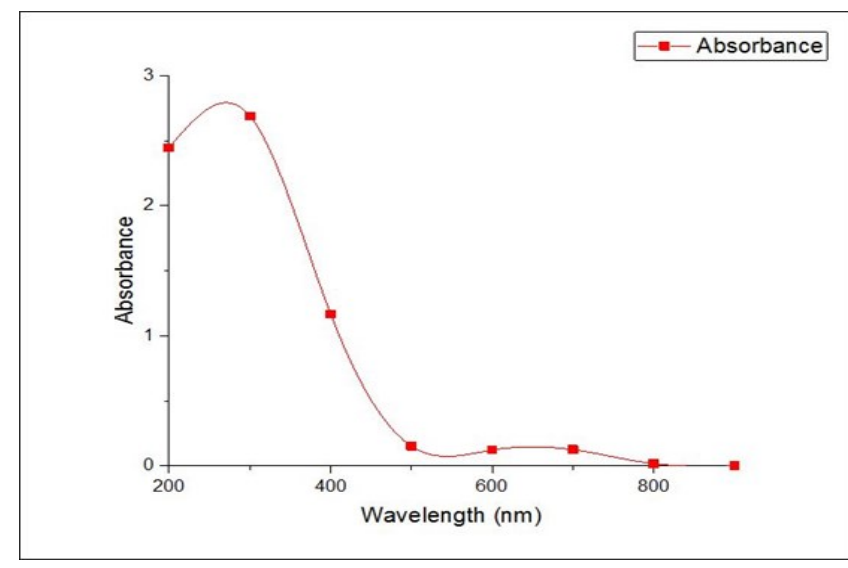

Fig. 1. UV-Vis spectra of grapheme oxide nanomaterial

and synergistic factor obtained with $1: 1$ and $1: 2$ combination of GO and malathion was $>20$ and $>1$, respectively, indicating the synergism of malathion with $\mathrm{GO}$ (Table 3). The overlapped d-m-r lines of $24 \mathrm{~h}$ larval exposure with $1: 1$ and $1: 2$ combinations of $M L+G O$ denotes no significant differences at $L C_{50}$ level of dose (Fig. 3) $(p>0.05)$.

\section{GO + Endosulphan (1:1 and 1:2)}

Bioassays with endosulphan combined with GO (1:1) increased the larvicidal efficacy of endosulphan by 1.38 -fold while larval exposure with GO + EN (1:2) increased the efficacy by 6.43 -fold (Table 4 ). The CTC (\% suppression) and SF obtained with both the combinations was $>20$ and $>1$, respectively (Table 4 ) denoting synergistic effects. The overlapping of the $d-m-r$ lines obtained with these mixtures denotes insignificant differences at $\mathrm{LC}_{50}$ level of dose (Fig. 4$)(p>0.05)$.

\section{Morphological aberrations in larvae}

Ae. aegypti larval exposure to GO+insecticide formulations at $\mathrm{LC}_{50}$ level showed visible morphological abnormalities. Exposure with graphene oxide and malathion (1:1 and 1:2) resulted in a deposition of black soot on

Table 2. Larvicidal efficacy of malathion and endosulphan against early fourth instars of Ae. aegypti after $24 \mathrm{~h}$ of exposure

\begin{tabular}{lll}
\hline \multirow{2}{*}{ Parameters } & \multicolumn{2}{c}{ Insecticides } \\
\cline { 2 - 3 } & Malathion & Endosulphan \\
\hline $\mathrm{LC}_{30}(\mathrm{mg} / \mathrm{L})$ & 0.046 & 0.180 \\
$95 \%$ Fiducial limits & $0.036-0.060$ & $0.124-0.263$ \\
$\mathrm{LC}_{50}$ (mg/L) & 0.063 & 0.338 \\
$95 \%$ Fiducial limits & $0.049-0.082$ & $0.232-0.492$ \\
LC 90 (mg/L) & 0.137 & 1.568 \\
$95 \%$ Fiducial limits & $0.105-0.178$ & $1.076-2.283$ \\
X $^{2}$ (df) & $0.877(4)$ & $0.158(5)$ \\
Slope \pm SE & $4.006 \pm 0.058$ & $2.001 \pm 0.083$ \\
\hline
\end{tabular}

\# $\mathrm{LC}_{30}$ - Lethal Concentration at which $30 \%$ larvae are killed, $\mathrm{LC}_{50}$ - Lethal Concentration at which $50 \%$ larvae are killed, LC 90 - Lethal Concentration at which $90 \%$ larvae are killed; $\mathrm{SE}=\mathrm{Standard}$ error. $\mathrm{X}^{2}=$ chi-square. $\mathrm{df}=$ degree of freedom. 


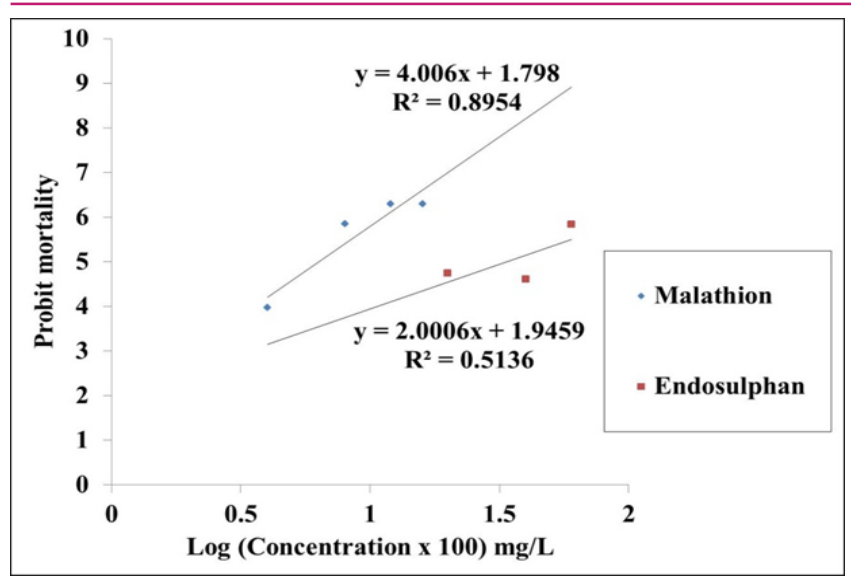

Fig. 2. Dosage-mortality regression lines on exposure of Ae. aegypti early fourth instars to the malathion and endosulphan for 24 hours.

the larval cuticle which may inhibit the cuticular transport of minerals and nutrients by diffusion (Fig. 5A; $5 B)$. On the other hand, disintegration of the gut viscera was observed in larvae exposed to $\mathrm{GO}$ and endosulphan (1:1 and 1:2) (Fig. 5C; 5D).

\section{Contact irritancy in adult females with insecticides alone}

Both the insecticide-impregnated papers induced a significant behavioral response in the female adults of $A e$. aegypti, higher irritancy was recorded in blood-fed adults in comparison to the unfed adults. The exposure to malathion and endosulphan could enable the fed mosquito to take first flight only after 4.67 and $5.00 \mathrm{sec}$,

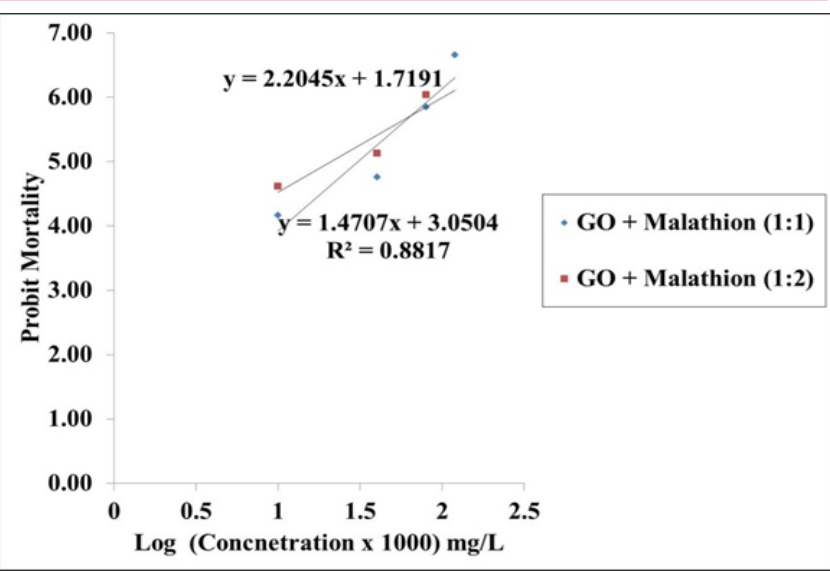

Fig. 3. Dosage-mortality regression lines on exposure of Ae. aegypti early fourth instars to the GO + ML (1:1 and 1:2) for 24 hours

and an average total of 32 and 40 take offs, respectively against control, inducing 5.64 and 8.56 relative irritability (Table 5).

\section{Synergistic contact irritancy studies with GO + in- secticides (1:1 and 1:2)}

\section{GO + Malathion (1:1 and 1:2)}

Addition of equal parts of GO and malathion increased the relative irritability of the unfed adult females to malathion by $28 \%$, whereas $1: 2$ synergized mixture raised the irritant effects by $32 \%$ (Table 6 ). In comparison to 4.67-5.67 take-offs in controls, 32.00-42.33 take-offs were recorded on exposure to $\mathrm{GO}+\mathrm{ML}$.

Table 3. Comparative larvicidal efficiency of malathion alone and synergized with graphene oxide (GO) in $1: 1$ and $1: 2$ ratios against early fourth instars of $A e$. aegypti after $24 \mathrm{~h}$ of exposure

\begin{tabular}{llll}
\hline \multirow{2}{*}{ Parameters } & Insecticide & \multicolumn{2}{c}{ GO + Malathion } \\
\cline { 2 - 3 } & Malathion & $1: 1$ & $\mathbf{1 : 2}$ \\
\hline $\mathrm{LC}_{30}(\mathrm{mg} / \mathrm{L})$ & 0.046 & 0.017 & 0.009 \\
$95 \%$ Fiducial limits & $0.036-0.060$ & $0.011-0.027$ & $0.005-0.017$ \\
$\mathrm{LC}_{50}(\mathrm{mg} / \mathrm{L})$ & 0.063 & 0.031 & 0.021 \\
$95 \%$ Fiducial limits & $0.049-0.082$ & $0.020-0.046$ & $0.011-0.037$ \\
LC 90 (mg/L) & 0.137 & 0.123 & 0.163 \\
$95 \%$ Fiducial limits & $0.105-0.178$ & $0.080-0.190$ & $0.090-0.294$ \\
$X^{2}$ (df) & $0.877(4)$ & $0.785(4)$ & $0.714(4)$ \\
Slope \pm SE & $4.006 \pm 0.058$ & $2.204 \pm 0.095$ & $1.471 \pm 0.083$ \\
Synergistic Ratio (SR) wrt LC 50 & - & 2.706 & 5.111 \\
Type of action (Based on SF) & - & Synergist & Synergist \\
Co-toxicity coefficient (CTC) wrt LC & & 30 & 40 \\
Type of action (Based on CTC) & - & Synergist & Synergist \\
\hline
\end{tabular}

\# $L C_{30}$ - Lethal Concentration at which $30 \%$ larvae are killed, $L C_{50}$ - Lethal Concentration at which $50 \%$ larvae are killed, LC $90-L e t h a l$ Concentration at which $90 \%$ larvae are killed; $S E=$ Standard error. $X^{2}=$ chi-square. $d f=$ degree of freedom. 
Table 4. Comparative larvicidal efficiency of endosulphan alone and synergized with graphene oxide (GO) in 1:1 and 1:2 ratios against early fourth instars of $\mathrm{Ae}$. aegypti after $24 \mathrm{~h}$ of exposure

\begin{tabular}{|c|c|c|c|}
\hline \multirow{2}{*}{ Parameters } & \multirow{2}{*}{ Endosulphan } & \multicolumn{2}{|c|}{ GO + Endosulphan } \\
\hline & & $1: 1$ & $1: 2$ \\
\hline $\mathrm{LC}_{30}(\mathrm{mg} / \mathrm{L})$ & 0.180 & 0.130 & 0.028 \\
\hline $95 \%$ Fiducial limits & $0.124-0.263$ & $0.070-0.152$ & $0.011-0.060$ \\
\hline $\mathrm{LC}_{50}(\mathrm{mg} / \mathrm{L})$ & 0.338 & 0.189 & 0.113 \\
\hline $95 \%$ Fiducial limits & $0.232-0.492$ & $0.125-0.270$ & $0.049-0.259$ \\
\hline $\mathrm{LC}_{90}(\mathrm{mg} / \mathrm{L})$ & 1.568 & 0.835 & 3.352 \\
\hline $95 \%$ Fiducial limits & $1.076-2.283$ & $0.567-1.229$ & $1.460-7.697$ \\
\hline$x^{2}(d f)$ & $0.158(5)$ & $0.865(5)$ & $0.437(5)$ \\
\hline Slope \pm SE & $2.001 \pm 0.083$ & $2.197 \pm 0.086$ & $1.471 \pm 0.083$ \\
\hline Synergistic Ratio (SR) wrt LC 50 & 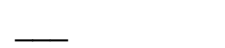 & 1.385 & 6.428 \\
\hline Type of action (Based on SF) & & Synergist & Synergist \\
\hline Co-toxicity coefficient (CTC) wrt $\mathrm{LC}_{50}$ & & 70 & 76 \\
\hline Type of action (Based on CTC) & & Synergist & Synergist \\
\hline
\end{tabular}

\# $L C_{30}$ - Lethal Concentration at which $30 \%$ larvae are killed, $L C_{50}$ - Lethal Concentration at which $50 \%$ larvae are killed, LC $90-L e t h a l$ Concentration at which $90 \%$ lanvae are killed; $S E=$ Standard error. $X^{2}=$ chi-square. $d f=$ degree of freedom.

Table 5. Response of 3-day old adult females (blood-fed and nonblood-fed) of Ae. aegypti to papers impregnated with insecticides and nanomaterials in the contact irritancy assays

\begin{tabular}{lccc}
\hline Test & $\begin{array}{l}\text { Mean Time lapse before first } \\
\text { take-off (in seconds) } \pm \text { SEM }\end{array}$ & $\begin{array}{l}\text { Mean number of take-off for } \\
\text { females (in 15 mins) } \pm \text { SEM }\end{array}$ & Relative irritability \\
\hline \multicolumn{4}{c}{ Malathion } \\
\hline Control & $1.33 \pm 0.67$ & $5.67 \pm 0.67$ & 1 \\
Non-blood-fed & $4.33 \pm 0.67$ & $23.33 \pm 1.86$ & 4.11 \\
Blood-fed & $4.67 \pm 0.33$ & $32.00 \pm 3.22$ & 5.64 \\
\hline \multicolumn{3}{c}{ Endosulphan } \\
Nontrol & $1.33 \pm 0.33$ & $4.67 \pm 0.34$ & 1 \\
Blood-fed & $4.00 \pm 0.58$ & $32.00 \pm 0.58$ & 6.85 \\
\hline
\end{tabular}

\section{GO + Endosulphan (1:1 and 1:2)}

Significant behavioural response in Ae. aegypti adult females was observed with binary mixture of GO+EN too (Table 6). GO+EN (1:1) increased the irritancy in mosquitoes by $24 \%$, whereas the combination of $1: 2$ resulted in higher irritant adults increasing the effects by $33 \%$ (Table 6), showing synergistic impact of GO. Similar to GO+ML, exposure to GO+EN caused 44.6753.33 flights in Ae. aegypti adult females as against 5.33-6.33 in controls.

\section{DISCUSSION}

Researchers are looking for environmentally sustainable and practical solutions to the breakout of Aedesborne diseases causing extensive human fatalities throughout the world. Reduction in mosquito larvae and, thus, the population is the major aim and target for efficient dengue vector control. For ages, various groups of chemical insecticides have been explored as mosquito control agents by eliminating mosquito life stages and limiting reproduction. Yet, their widespread usage has harmed our environment, non-target animals and human health. As a result, scientists are developing and testing nanomaterials as insecticide protectants and transporters in order to develop and employ these nanoinsecticides as effective and environmentally friendly mosquito management options. A few studies have explored graphene oxide as the possible nanomaterial which could be coupled with insecticides to formulate nanopesticides against insect pests and other harmful organisms (Das et al., 2021; Nehra et al., 2021; Raj et al., 2021).

Thus, the possible synergism of malathion and endo- 
Gupta, D. et al. / J. Appl. \& Nat. Sci. 13(4), 1265 - 1273 (2021)

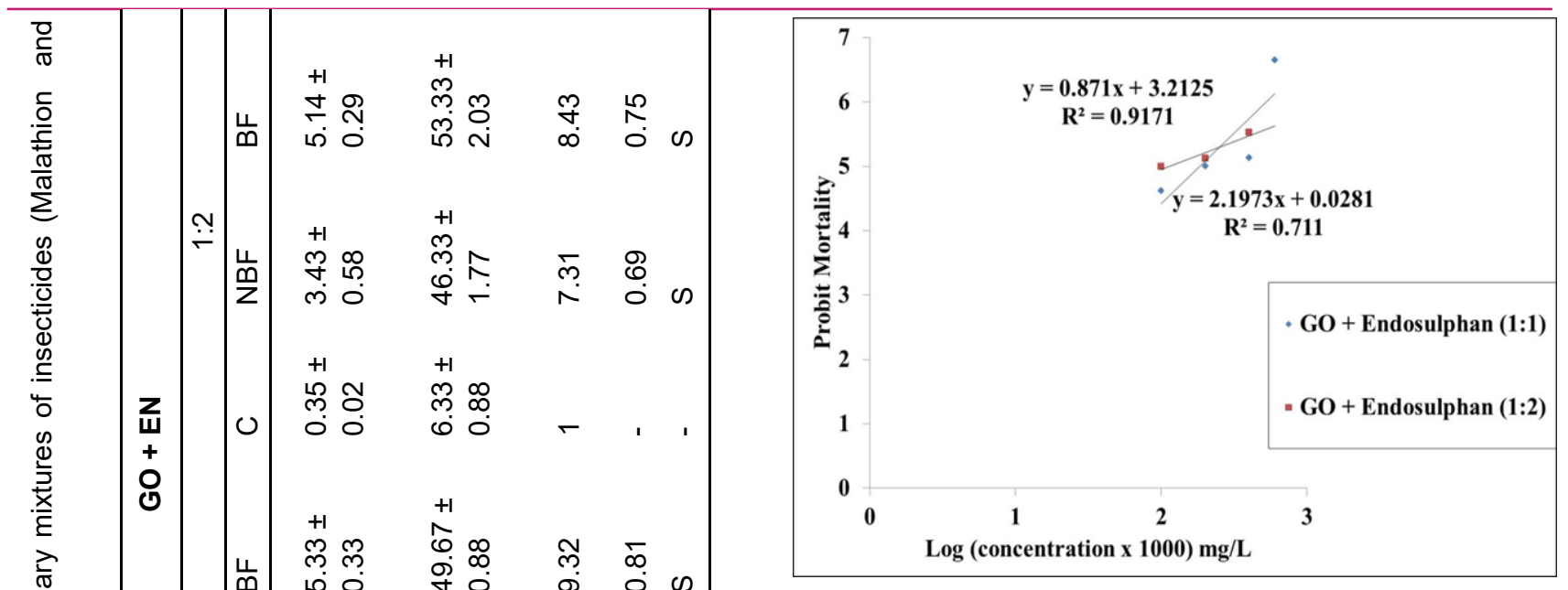

Fig. 4. Dosage-mortality regression lines on exposure of Ae. aegypti early fourth instars to the GO + EN (1:1 and 1:2) for 24 hours.

sulphan with GO was assessed in the current study. The formulations were investigated for their toxic and irritant effects on an Indian strain of $A e$. aegypti with the goal of controlling dengue larvae and adults with minimal use of environmentally hazardous insecticides.

The study showed that conjugating graphene oxide with malathion and endosulphan improved the efficacy of both the pesticides against early fourth instars of $A e$. aegypti. Remarkably, the GO+insecticide in 1:2 ratio decreased the LC values significantly as compared to insecticides alone and GO+insecticide (1:1) despite the increase in the toxic component of the mixture. The $\mathrm{GO}+\mathrm{ML}$ combinations were found to more toxic than GO+EN combinations recommending their use against larval stages of $A e$. aegypti as shown in Tables 3 and 4). Similar results were reported by Wang et al. (2019b) against Asian corn borer, Ostrinia furnacalis. They recorded $\mathrm{LC}_{50}$ values of 1.96 and $4.23 \mu \mathrm{g} / \mathrm{mL}$ respectively with exposure to monosultap and imidacloprid, which reduced to 1.29 and $2.31 \mu \mathrm{g} / \mathrm{mL}$ after subjection to Monosultap+GO and Imidacloprid+GO combinations.

A few studies have shown that combining the active bioactive constituent with nanomaterials could not only improve the efficacy of toxicants against target species but also reduced volatility, improved the stability of the active molecules, and provided a longer-lasting release, resulting in improved insecticide protection with low doses (Chaudhari et al., 2021; Xiao et al., 2021). The application of encapsulated garlic essential oil in polyethylene glycol (PEG) to harvested rice infested with red flour beetles (Tribolium castaneum) resulted in $80 \%$ increased mortality with the nanomaterials formulation, as compared to only $11 \%$ recorded with essential oil alone (Yang et al., 2009). Similarly, a declined feeding was observed in Spodoptera litura and Achaea janata larvae when fed upon castor leaves treated with pinene and linalool-encapsulated silica dispersed in acetone thus leading to death owing to malnutrition 


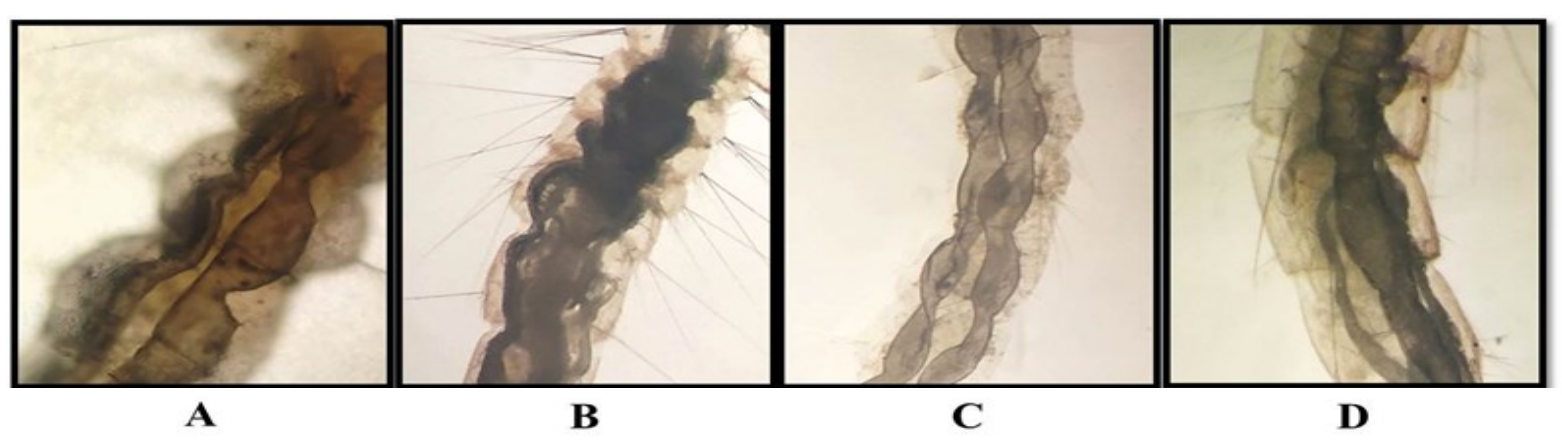

Fig. 5. Ae. aegypti larvae showing deposition of black soot on subjection to (A) $G O+M L(1: 1) ;(B) G O+M L(1: 2) ;(C)$ $G O+E N(1: 1) ;$ and (D) GO+EN (1:2)

(Rani et al., 2014). In contrast, in field trials, sodium alginate-encapsulated imidacloprid was equally efficient against leaf hopper as imidacloprid alone (Kumar et al., 2014). In a separate field trial, Brassica chinese treated with silica-encapsulated chlorfenapyr for three days achieved equivalent or better control of the diamondback moth (Plutella xylostella) (Song et al., 2012).

The study revealed morphological aberrations in the larvae (Fig. 5) and significantly higher contact irritancy in the adult females of Ae. aegypti on exposure to GOinsecticide combinations (Table 6). The higher irritancy in blood-fed females was observed in comparison to the unfed females, though insignificant. Such studies have not been conducted yet against mosquitoes or any other organism. However, GO combined with chlorpyrifos, pyridaben and $\beta$-cyfluthrin induced higher contact toxic effects in $T$. urticae $(1.78,1.75$ and 1.50 fold) and T. truncatus; (1.55, 1.56 and 1.77-fold), respectively (Wang et al., 2019a). The synergism of $\beta$ cyfluthrin, monosultap and imidacloprid with GO against Asian corn borer induced 2.10, 1.51 and 1.83fold higher contact toxicity in comparison to the respective individual (Wang et al., 2019b).

It is well recognized that the delayed release of active compounds of pesticides can reduce toxicity. Several researches have looked into whether continual pesticide release from nanomaterials could lessen toxicity. The cytotoxicity of imidacloprid loaded onto sodium alginate nanoparticles demonstrated that the concentration loaded-pesticide nanoformulation was less hazardous than the original pesticide, albeit by a tiny margin (Kumar et al., 2014).

The current study is significant because it not only reduces the amount of pesticide used, but it also makes the conjugate mixture more toxic, cost-effective, and environment-friendly. Combining nanomaterials with pesticides to boost larvicidal and irritating efficiency of insecticides against $A$ e. aegypti could give a long-term and eco-safe mosquito control strategy. The multifunctional mechanism that allows $\mathrm{GO}$ to act as a synergist for existing pesticides reveals the high potential for its use in vector control. This research allows to look at the use of nanotechnology in the management of the dengue vector, as nanomaterials have the potential to reduce the adverse effects of insecticides.

\section{Conclusion}

The present study concluded that the outcome of the efficacy of GO-insecticide (malathion and endosulphan) combinations against the Indian strain of the dengue vector Ae. aegypti, with a proposition, will help combat the dengue hazard and develop a strategy to counter insecticide resistance in mosquitoes. The investigations on the contact irritant and larvicidal capabilities of synergistic combinations of GO with malathion and endosulphan against $A e$. aegypti revealed more effective insecticidal action on $A e$. aegypti, recommending the development of graphene-based insecticidal nanoformulations against target pests. The field trials with these formulations would help in the standardization of formulation dosage and mode of application against mosquitoes to fight their menace. We believe that the outcome of the study would be useful to combat the dengue hazard and in devising a strategy to deal with the problem of insecticide resistance in mosquitoes.

\section{ACKNOWLEDGEMENTS}

The authors are highly grateful to the Principal, Acharya Narendra Dev College for providing infrastructure and research facilities. The research was supported by Acharya Narendra Dev College, University of Delhi, Delhi

\section{Conflict of interest}

The authors declare that they have no conflict of interest.

\section{REFERENCES}

1. Abbott W.S. (1925). A method of computing the effectiveness of an insecticide. J. Econ. Entomol. 18, 265-267.

2. Chaudhari, A.K., Singh, V.K., Kedia, A., Das, S. \& Dubey, N.K. (2021). Essential oils \& their bioactive compounds as eco-friendly novel green pesticides for management of 
Gupta, D. et al. / J. Appl. \& Nat. Sci. 13(4), 1265 - 1273 (2021)

storage insect pests: prospects \& retrospects. Environ. Sci. Pollut. Res. 28, 18918-18940. https:// doi.org/10.1007/s11356-021-12841-w

3. Chen, J., Yao, B., Li, C. \& Shi, G. (2013). An improved Hummers method for eco-friendly synthesis of graphene oxide. Carbon. 64, 225-229. https://doi.org/10.1016/ j.carbon.2013.07.055

4. Das, K., Jhan, P.K., Das, S.C., Aminuzzaman, F.M. \& Ayim, B.Y. (2021). Nanotechnology: Past, Present \& Future Prospects in Crop Protection. IntechOpen, DOI: 10.5772/intechopen.98703. Available from: https:// www.intechopen.com/online-first/77333

5. Dixit, S., Ahsan, H \& Khan, F.H. (2021). Pesticides \& plasma proteins: unexplored dimensions in neurotoxicity. Internat. J. Pest Manage. 2021,1-10. https://doi.or $\mathrm{g} / 10.1080 / 09670874.2021 .1917725$

6. Gautam, D., Lal, S \& Hooda, S. (2020). Adsorption of rhodamine $6 \mathrm{~g}$ dye on binary system of nanoarchitectonics composite magnetic graphene oxide material. J. Nanosci. Nanotechnol. 20, 2939-2945. https://doi.org/10.1166/ jnn.2020.17442

7. Gupta, A., Samal, R.R \& Kumar, S. (2021). Physiological $\&$ reproductive fitness cost in Aedes aegypti on exposure to toxic xenobiotics in New Delhi, India. J. Appl. Nat. Sci. 13, 71-78. https://doi.org/10.31018/jan s.v13i1.2470

8. Jampílek J., Král'ová K. \& Fedor P. (2020). Bioactivity of nanoformulated synthetic and natural insecticides and their impact on environment. In: Fraceto L.F., S.S. de Castro V.L., Grillo R., Ávila D., Caixeta Oliveira H., Lima R. (eds) Nanopesticides. Springer, Cham, pp 165 -225. https://doi.org/10.1007/978-3-030-44873-8_7

9. Kalyanasundaram, M \& Das, P.K. (1985). Larvicidal \& synergistic activity of plant extracts for mosquito control. Ind. J. Med. Res. 82, 19-23.

10. Kumar, S., Bhanjana, G., Sharma, A., Sidhu, M.C \& Dilbaghi, N. (2014). Synthesis, characterization \& on field evaluation of pesticide loaded sodium alginate nanoparticles. Carbohydr. Polym. 101,1061-1067. https://doi.org/1 0.1016/j.carbpol.2013.10.025

11. Lu, Z., Zhang, C.,Gao, Y., Wang, W., Lin, J \& Du, F. (2021). Simple, effective, \& energy-efficient strategy to construct a stable pesticide nanodispersion. ACS Agric. Sci. Technol. 1, 329-337. https://doi.org/10.1021/acsa gscitech.1c00018

12. Monteiro, R.A., Camara, M.C., de Oliveira, J.L., Campos, E.V.R., Carvalho, L.B., de Freitas Proença, P.L., GuilgerCasagre, M., Lima, R., do Nascimento, J., Gonçalves, K.C. \& Polanczyk, R.A. (2021). Zein based-nanoparticles loaded botanical pesticides in pest control: An enzyme stimuli-responsive approach aiming sustainable agriculture. J. Hazard. Mater. 417, p.126004. https://doi.org/10.1 016/j.jhazmat.2021.126004

13. Nehra, M., Dilbaghi, N., Marrazza, G., Kaushik, A., Sonne, C., Kim, K.H. \& Kumar, S. (2021). Emerging nanobiotechnology in agriculture for the management of pesticide residues. J. Hazard. Mater. 401, p.123369. https:// doi.org/10.1016/j.jhazmat.2020.123369

14. Raj, S.N., Anooj, E.S., Rajendran, K. \& Vallinayagam, S. (2021). A comprehensive review on regulatory invention of nano pesticides in Agricultural nano formulation \& food system. J. Mol. Struct. 1239, 1-9. https://doi.org/10.1016/ j.molstruc.2021.130517
15. Rani, P.U., Madhusudhanamurthy, J \& Sreedhar, B. (2014). Dynamic adsorption of pinene \& linalool on silica nanoparticles for enhanced antifeedant activity against agricultural pests. J. Pest Sci. 87, 191-200. https:// doi.org/10.1007/s10340-013-0538-2

16. Samadi, S \& Abbaszadeh, M. (2017). Synthesis \& characterization of MgO/PEG/GO nanocomposite \& its application for removal of copper (II) from aquatic media. Bull. Soc. R. Sci. Liège. 86, 271-280. 10.25518/00379565.6709

17. Samal R.R \& Kumar, S. (2021). Cuticular thickening associated with insecticide resistance in dengue vector, Aedes aegypti L. Internat. J. Trop. Insect Sci. 41, 809-820. https://doi.org/10.1007/s42690-020-00271-z

18. Samal R.R. \& Kumar S. (2018). Susceptibility status of Aedes aegypti L. against different classes of insecticides in New Delhi, India to formulate mosquito control strategy in fields. The Open Parasitol. J. 6, 52-62. 10.2174/1874421401806010052

19. Samal, R.R., Panmei, K., Lanbiliu, P \& Kumar, S. (2020). Biochemical characterization of acetamiprid resistance in laboratory-bred population of Aedes aegypti L. larvae. Proceedings of the International Conference \& the 10th Congress of the Entomological Society of Indonesia (ICCESI 2019), Bali, Indonesia. 8, 169-176. https:// doi.org/10.2991/absr.k.200513.030

20. Song, M.R., Cui, S.M., Gao, F., Liu, Y.R., Fan, C.L., Lei, T.Q \& Liu, D.C. (2012). Dispersible silica nanoparticles as carrier for enhanced bioactivity of chlorfenapyr. J. Pestic. Sci. 37, 258-260, https://doi.org/10.1584/jpestics.D12-027

21. Trisyono, A \& Whalon, M. E (1999). Toxicity of neem applied alone \& in combination with Bacillus thuringiensis to Colorado potato beetle (Coleoptera: Chrysomelidae). Journal of Economic Entomology, 92: 1281-1228. https://doi.org/10.1093/jee/92.6.1281

22. Wang, X., Xie, H., Wang, Z \& He, K. (2019a). Graphene oxide as a pesticide delivery vector for enhancing acaricidal activity against spider mites. Colloids Surf. B: Biointerface. 173, 632-638. https://doi.org/10.1016/j.colsurfb.2 018.10 .010

23. Wang, X., Xie, H., Wang, Z., He, K \& Jing, D. (2019b). Graphene oxide as a multifunctional synergist of insecticides against lepidopteran insect. Environ. Sci.: Nano. 6,75-84. 10.1039/C8EN00902C

24. World Health Organization (2016). Monitoring \& managing insecticide resistance in Aedes mosquito populations. http:// apps.who.int/iris/bitstream/handle/10665/204588/ WHO_ZIKV_VC_16.1_eng.pdf?sequence $=2$

25. Xiao, Z., Zhang, Y., Niu, Y., Ke, Q. \& Kou, X. (2021). Cyclodextrins as carriers for volatile aroma compounds: A review. Carbohydr. Polym. p.118292. https://doi.org/10.10 16/j.carbpol.2021.118292

26. Yang, F.L., Li, X.G., Zhu, F \& Lei, C.L. (2009). Structural characterization of nanoparticles loaded with garlic essential oil \& their insecticidal activity against Tribolium castaneum (Herbst)(Coleoptera: Tenebrionidae). J. Agric. Food Chem. 57, 10156-10162. https://doi.org/10.1021/ff902 3118

27. Zhang, P., Guo, Z., Ullah, S., Melagraki, G., Afantitis, A. \& Lynch, I. (2021). Nanotechnology \& artificial intelligence to enable sustainable \& precision agriculture. Nature Plants, 7, 864-876. https://doi.org/10.1038/s41477-021-00 946-6 